\title{
HabEx space telescope exoplanet instruments
}

Stefan Martin, Mayer Rud, Dimitri Mawet, Joel Nissen, Stuart Shaklan, et al.

Stefan Martin, Mayer Rud, Dimitri Mawet, Joel Nissen, Stuart Shaklan, Luis Marchen, "HabEx space telescope exoplanet instruments," Proc. SPIE 10698, Space Telescopes and Instrumentation 2018: Optical, Infrared, and Millimeter Wave, 106980T (21 August 2018); doi: 10.1117/12.2314410

Event: SPIE Astronomical Telescopes + Instrumentation, 2018, Austin, Texas, United States 


\title{
HabEx Space Telescope Exoplanet Instruments \\ Stefan Martin, Mayer Rud, Dimitri Mawet*, Joel Nissen, Stuart Shaklan, Luis Marchen, Jet Propulsion Laboratory, California Institute of Technology, Pasadena, CA 91109. \\ * California Institute of Technology, Pasadena, CA 91125.
}

\begin{abstract}
The HabEx (Habitable Exoplanet) space telescope mission concept carries two complementary optical systems as part of its baseline design, a coronagraph and a starshade, that are designed to detect and characterize planetary systems around nearby stars. The starshade is an external occulter which would be $72 \mathrm{~m}$ in diameter and fly some 124,000 km ahead of the telescope. A starshade instrument on board the telescope enables formation flying to maintain the starshade within $1 \mathrm{~m}$ of the line of sight to the star. The starshade instrument has various modes, including imaging from the near UV through to the near infrared and integral field spectroscopy in the visible band. The coronagraph would provide imaging and integral field spectroscopy in the visible band and would reach out to $1800 \mathrm{~nm}$ for low resolution spectroscopy in the near infrared. To provide the necessary stability for the coronagraph, the telescope would be equipped with a laser metrology system allowing measurement and control of the relative positions of the principal mirrors. In addition, a fine guidance sensor is needed for precision attitude control. The requirements for telescope stability for coronagraphy are discussed. The design and requirements on the starshade will also be discussed.
\end{abstract}

Keywords: HabEx, space telescope

\section{INTRODUCTION}

The HabEx telescope ${ }^{1}$ is a three mirror anastigmat design (TMA) with a 4-m diameter primary mirror, 2.5-m off axis. A $4 \mathrm{~m}$ primary mirror (M1) directs light to the secondary (M2) then on to the tertiary (M3), producing a collimated $50 \mathrm{~mm}$ beam at the output. Figure 1 shows the telescope's optical layout. Instruments are arranged near the tertiary. In the annular field TMA design, the Cassegrain focus is spatially larger than its exit pupil and this widely separates the beams for each field of view at the tertiary mirror, facilitating the extraction of light for the individual instruments. The telescope's output beam is directed back towards the secondary mirror and by placing a mirror between the tertiary and the TMA exit pupil, an individual field can be extracted and passed to an instrument. This design allows for different optical coatings at different locations on the tertiary, or separate tertiary mirrors with instrument-specific coatings, to aid transmission efficiency with some instruments. The general astrophysics instruments, a high resolution UV spectrograph (UVS) ${ }^{1,2}$ and a general purpose camera/spectrograph, the HabEx Workhorse Camera (HWC) ${ }^{1}$ require performance down to $115 \mathrm{~nm}$ in the UV, so a protected aluminum coating is required on at least the first two mirrors. The four science instruments are arranged on the side of the telescope near M3 as shown in Figure 1. An alternative often employed, particularly with onaxis telescope designs, is to place the instruments behind the primary mirror, above the bus. However, side mounting allows easier extraction of the instrument modules for servicing (a NASA requirement) and takes advantage of existing

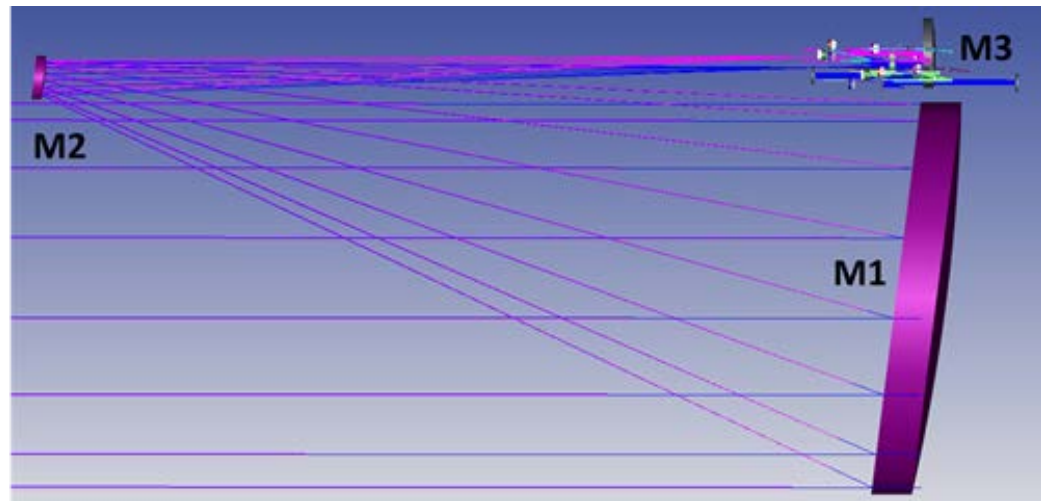

Figure 1: Telescope optical layout. The instruments area arranged near M3. volume created by the off-axis design, notably the presence of the secondary and tertiary mirrors which are offset beside the primary. Furthermore, it creates easy access to radiators needed for cooling the detectors. Rear mounting would make use of space behind the primary, between its supporting structure and the bus, but may increase the overall telescope spacecraft height. Cooling paths would generally be longer and extraction of individual modules for servicing might be more complex. However, both design concepts are viable and would occupy similar volumes. 


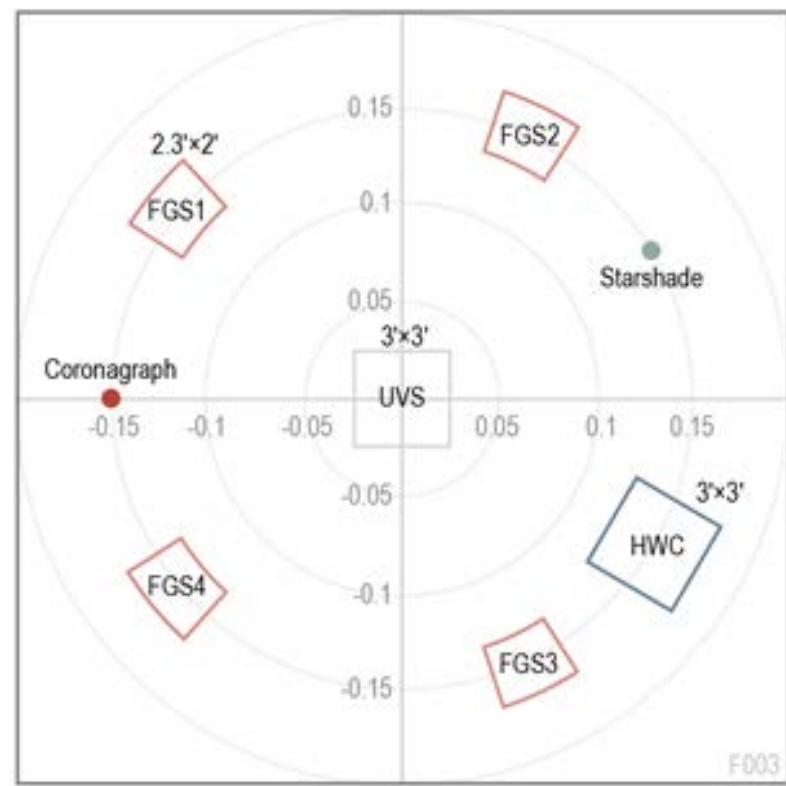

Figure 2: Fields of view showing the four science instruments and the fine guiding system areas.
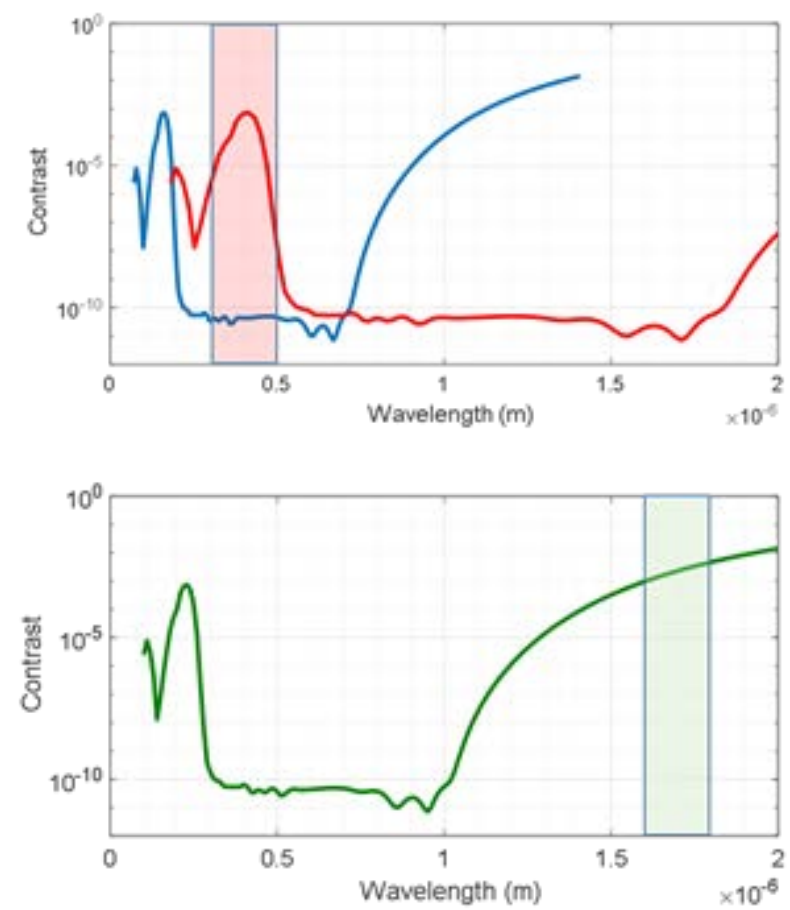

Figure 3: Starshade transmission functions. Lower chart: the visible band showing deep contrast between 300 and 1,000 nm. On the right is shown the guide band; the infrared sensor detects the leakage light between 1600 and $1800 \mathrm{~nm}$. Upper chart: showing the IR science band extending from $600 \mathrm{~nm}$ to $1800 \mathrm{~nm}$ and its guide band from 300 to $450 \mathrm{~nm}$, detected on the UV guide channel. Also shown is the UV science band. Its guide band will be in the IR, as shown in the lower chart.

\section{INSTRUMENTS}

Figure 2 shows the fields of view of the four instruments plus areas in the field used for fine guiding. The UVS occupies the center of the field, having a 3'x3' field of view, and the coronagraph views a small field to the left. The starshade instrument views a small field to the right and slightly upwards while the HWC views a wide area to the right and slightly downwards. After striking the tertiary, the rays are collimated and converge towards a common on-axis pupil plane. Before reaching that plane however, the beams are extracted by fold mirrors and directed to steering mirrors at the pupil plane. These steering mirrors direct the light into the instruments or the fine guidance sensors. An exception is the UVS, which has its own tertiary sending the light directly to a diffraction grating and is thereby extracted with the minimum number of folds in order to preserve optical throughput.

\subsection{Starshade Instrument Overview}

Starshade operation requires both science and formation flying cameras to view the starshade simultaneously. A high suppression, extremely dark shadow exists behind the starshade and the telescope is placed as far back as possible within this shadow $(\sim 124,000 \mathrm{~km})$ while maintaining high starlight suppression. The telescope can move laterally $\pm 1 \mathrm{~m}$ within the shadow and the tips of the starshade form an angle of 60 mas to the line of sight when operating in the 300 to $1,000 \mathrm{~nm}$ spectral band. The HabEx starshade is of the numerically optimized type producing a high suppression over a designed wavelength band. Light of both shorter and longer wavelengths is attenuated, but leaks into the shadow and is used for starshade position determination. Figure 3 shows the starshade transmission functions for the three planned science bands, 200 to $667 \mathrm{~nm}, 300$ to $1,000 \mathrm{~nm}$, and 540 to $1,800 \mathrm{~nm}$. When performing science at longer "red" wavelengths, shorter wavelength "blue" light is used for guiding and vice versa.

To obtain suppression down to $200 \mathrm{~nm}$ in the UV, the starshade can be moved further away from the telescope, potentially achieving an IWA of 40 mas. For infrared science, the starshade moves closer to the telescope and the IWA will increase in proportion to the wavelength. Table 1 shows the science bands, ranges and IWAs. The starshade instrument, shown schematically in Figure 4, contains six beam paths to accommodate three optical channels: UV, visible, and infrared. Light entering the starshade camera is split by dichroic optics into UV, then visible and IR beam paths, so all of these channels can be operated simultaneously. Camera and spectrograph properties are shown in Table 1. The UV channel carries a 
simple slit spectrograph employing a grism with $\mathrm{R}=7$. The visible channel carries a broadband integral field spectrograph (IFS) capable of covering the wavelength range from 450 to $1,000 \mathrm{~nm}$, plus an imaging camera for more rapid and wide-field system imaging. The infrared channel carries an IFS with $\mathrm{R}=40$ to enable disc and object spectroscopy.

\section{Visible Channel}

The visible channel is the principal science channel and carries a camera and an integral field spectrograph (IFS). The layout is shown schematically in Figure 5. Light from the telescope M3 strikes the fold mirror and then the FSM at the entrance to the starshade instrument. It then passes through a dichroic optic, which reflects UV light. The remaining visible and infrared light passes to a second dichroic where the visible light is reflected to an off-axis paraboloidal mirror and thence to a focus where field stops are inserted to limit the field of view, one for the imaging mode (11.9" diameter) and a second for spectroscopy (1.9" diameter). This focus is reimaged by an ellipsoidal mirror to the focal plane. A filter wheel is inserted after the ellipse with filters to select wavebands appropriate for different starshade ranges. For example, with the starshade at the nominal range for visible work, the filter would pass 450 to $1,000 \mathrm{~nm}$ light. With the starshade more distant as set up for UV science, the range would be 450 to $667 \mathrm{~nm}$ (see Table 2). Further filters and polarizing optics for polarization studies could also be inserted here such as the science filters provided for coronagraphy. The imaging focal plane consists of a single EMCCD operated at 153K. The chosen type is a modified CCD201 with delta doping and a thickened substrate together with a broadband "astro" coating giving response out to $1000 \mathrm{~nm}$. Pixel scale is as shown in Table 1. During a thruster firing, the thruster plumes scatter sunlight towards the telescope, raising the background level significantly so the sensor is read out at $1 \mathrm{kHz}$ to keep the accumulated photon count well below full well.

For spectroscopy, a powered mirror is inserted in the beam following the paraboloid, producing a large increase in the F\# from 47 to 1330 . Via a fold mirror, this beam is focused onto a microlens array (MLA) which forms the entrance to the IFS. The IFS consists of the MLA, a matching multiple aperture mask to restrict stray light, a set of lenses to collimate the beam, prisms to disperse the wavelengths and a second set of lenses to focus onto the focal plane. This type of IFS is described in McElwain et al. ${ }^{3}$. Its operation can be visualized thus: for each microlens array element, a spectrum is produced on the focal plane. The optical geometry involving the MLA ensures that the spectra do not overlap or interfere with each other. An image can be formed of the scene at one wavelength by using all the pixels on the focal plane that correspond to the same wavelength. A series of images known as "slices" can be assembled into a "data cube" with sides corresponding to the directions of the field of view and height corresponding to wavelength. Thus, the scene is reproduced in a stack of images representing narrow wavelength bands. To calibrate the images, it is necessary to provide a calibration source with at least one known wavelength in the band and that illuminates
Table 1: Starshade instrument parameters.

\begin{tabular}{|l|c|c|c|}
\hline \multicolumn{1}{|c|}{ Science Bands } & UV & Visible & IR \\
\hline Wavelength range (nm) & $200-667$ & $300-1,000$ & $540-1,800$ \\
\hline Starshade range (Mm) & 186 & 124 & 69 \\
\hline IWA (mas) & 40 & 62 & 108 \\
\hline Guide band (nm) & $1,600-1,800$ & $1,600-1,800$ & $300-450$ \\
\hline \multicolumn{1}{|c|}{ Cameras } & UV Channel & Visible & IR (Guide) \\
\hline FOV & $10.2^{\prime \prime}$ & $11.9^{\prime \prime}$ & - \\
\hline Wavelength bands & $200-450 \mathrm{~nm}$ & $\begin{array}{c}450-1,000 \\
\text { nm }\end{array}$ & $\begin{array}{c}950-1,800 \\
\text { nm }\end{array}$ \\
\hline Pixel resolution & 14.2 mas & 14.2 mas & $12 \mathrm{~cm}$ \\
\hline Telescope resolution & 21 mas & 21 mas & - \\
\hline IWA (at longest $\Lambda$ ) & 40 mas & 62 mas & - \\
\hline Detector & $1 \times 1$ & $1 \times 1$ & $1 \times 1$ \\
& CCD201 & CCD201 & LMAPD \\
\hline Array width (pixels) & 1024 & 1024 & 256 \\
\hline Spectrometers & UV Channel & Visible & IR \\
\hline FOV & $10.2 "$ & $1.9 "$ & $3.8 "$ \\
\hline Wavelength bands (nm) & $200-450$ & $450-1000$ & $950-1,800$ \\
\hline $\begin{array}{l}\text { Spectrometer } \\
\text { resolution }\end{array}$ & 7 & 140 & 40 \\
\hline Spectrometer type & Slitgrism & IFS & IFS \\
\hline Detector & $1 \times 1$ CCD201 & $1 \times 1$ CCD282 & $2 \times 2$ \\
\hline Array width (pixels) & 1024 & 4,096 & 2,048 \\
\hline
\end{tabular}


the entire MLA. The calibration source light is injected when required through the fold mirror, which has a small leakage $\sim 2 \%$.

The IFS's focal plane consists of a large single electron multiplying CCD (Teledyne/e2v CCD282) operated at $163 \mathrm{~K}$. This is a modified version of the off-the-shelf item with delta doping and a thickened substrate together with a broadband "astro" coating giving response out to $1,000 \mathrm{~nm}$. The device format is an $8 \mathrm{k} \times 4 \mathrm{k}$ array with frame store areas at both sides of the 8k length, and a $4 \mathrm{k} \times 4 \mathrm{k}$ center imaging area. The spectral images are produced on the $4 \mathrm{k}$ square center area and moved into the frame stores before readout at high EMCCD gain. Again, during a thruster firing, the sensor is read out as fast as possible to keep the accumulated photon count well below full well.

\section{Ultraviolet Channel}

This channel (Figure 6) carries a low-resolution spectrometer and is also used as the guide channel for IR science. Light from the telescope M3 strikes the fold mirror and then the FSM at the entrance to the starshade instrument. It then reaches a dichroic optic that reflects the UV light to an off-axis paraboloidal mirror and thence through a field stop to an ellipsoidal mirror. Following the ellipse is a filter wheel to allow filter selection as shown in Table 2. Two field stops are provided, one to allow a field of view up to 10.2" diameter, and another with 0.02 " diameter to select individual objects. The field stops are mounted on a piezoelectric stage to allow selection and positioning. The beam is then refocused to the focal plane, passing through a filter placed at the intermediate exit pupil which removes light of wavelengths longer than $450 \mathrm{~nm}$. Also at this exit pupil, a grism can be introduced for low-resolution spectroscopy. With the grism removed, the camera forms an undispersed image. With the introduction of a mirror further downstream, the exit pupil is relayed to the focal plane, forming a pupil image suitable for starshade guiding. The pupil scale need not be large; a $32 \times 32$ pixel image is formed with each pixel covering a $12 \mathrm{~cm}$ square section of the entrance pupil. The focal plane consists of a single EMCCD (CCD201) operated at 153K. The chosen type is a modified version of the off-the-shelf item optimized for high UV sensitivity by deep depletion and delta-doping processes ${ }^{4}$, together with a broad band coating to improve response down to $200 \mathrm{~nm}$. Pixel scale

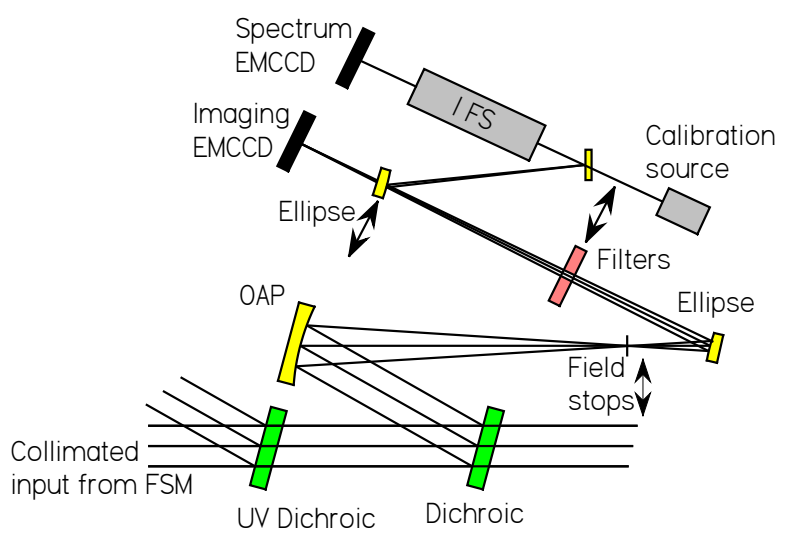

Figure 5: Schematic layout of starshade visible channel.

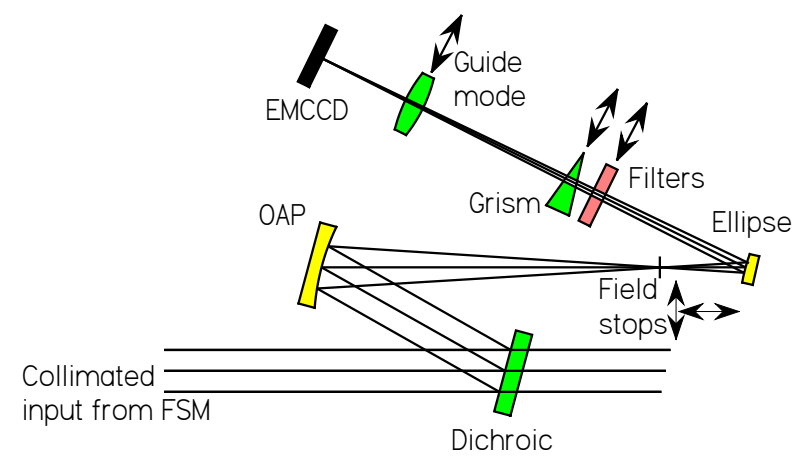

Figure 6: Schematic layout of starshade UV channel. 
is as shown in Table 1 . The format is a $1 \mathrm{k} \times 1 \mathrm{k}$ array with adjacent frame store. Again, the CCD will be read out with high gain to minimize read noise and during a thruster firing, the sensor is read out at $1 \mathrm{kHz}$ to keep the accumulated photon count down.

\section{Infrared Channel}

The infrared channel is the primary guide channel used for both visible and UV science, and also carries an infrared IFS. When the IFS is being used, guiding is on the UV guide channel. Infrared light entering the instrument passes through both dichroics and is reflected off a paraboloidal mirror (Figure 7). A filter wheel operates to allow band selection as shown in Table 1. At

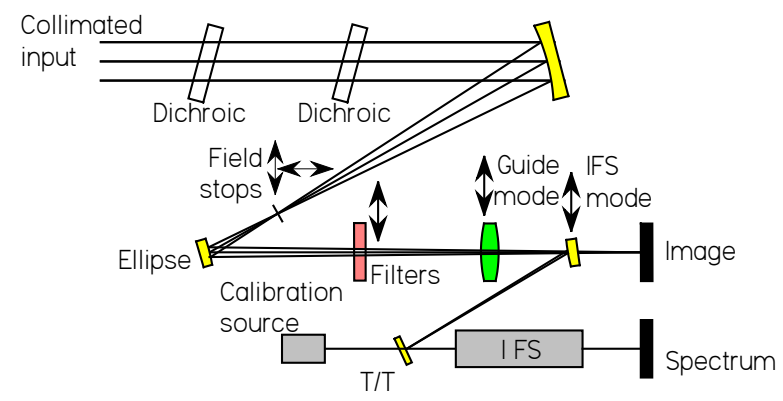

Figure 7: Schematic layout of starshade IR channel the focus, a field stop limits the field of view to $4 "$ for the IFS, slightly larger than the IFS MLA FOV.

The guide channel consists of a lens to relay the exit pupil following the ellipsoid to the focal plane with the magnification providing 32 pixels across the telescope aperture. The focal plane consists of a single linear mode avalanche photodiode (LMAPD) array detector based on a HgCdTe sensor (Saphira array by Selex). The avalanche gain-mode allows the effective read noise to be reduced (but not yet to the extent possible in EMCCDs). The detector is cooled to 60K to minimize dark current. Note that an IR imaging mode is easily provided in this layout, though not called for in the STM. The science channel consists of a powered relay mirror inserted near the guide channel relay lens. This provides the necessary larger focal length to the MLA. Before reaching the MLA, the beam is folded at a plane mirror. A calibration source is provided behind this mirror, injecting through it, so that the position of the spectrum can be identified on the IFS focal plane. The IFS utilizes a proposed variant of the Saphira detector with a $1 \mathrm{k} \times 1 \mathrm{k}$ format and smaller pixels (12 micron) operated at $60 \mathrm{~K}$. Four of these detectors are arrayed in a $2 \times 2$ format to provide a full FOV of $3.8 "$ at R= 40 . The IFS optics follow the same general design as for the visible IFS, with appropriate optical prescription changes. The field of view is Nyquist sampled by the lenslets and likewise, the spectrum is Nyquist sampled at the detector. During a thruster firing, due to its parallel output format and windowing capability, the sensor can be read out at very high frame rates to keep the accumulated charge down and thus avoid contamination of the science data by charge persistence.

\section{The Starshade}

The $72 \mathrm{~m}$ diameter starshade ${ }^{5}$ flies in formation with the telescope, creating a deep shadow that suppresses the light from the parent star and thereby reveals the reflected light from its exoplanets. The optical design and position of the starshade occulter shape, along with the resolution and performance of the telescope and starshade instrument, determines the depth of the contrast in the dark field. The size of the starshade occulter necessitates a deployable architecture that is passively shape controlled, both mechanically and thermally. The starshade mechanical system is designed to reliably deploy on orbit and meet the specified shape accuracy, shape stability, and solar glint requirements. The selected solution for mechanical deployment is the "furled petal" architecture in which the stowed petals are wrapped around a central drum. The starshade occulter must also be mobile, since it must be positioned and maintained accurately on the LOS of the telescope to the target star. With a separation of $124,000 \mathrm{~km}$ from the telescope, the starshade spends up to $80 \%$ of the mission slewing from one target to the next.

\section{Starshade Guiding}

The ultraviolet and infrared channels have guide camera modes, which project a pupil image onto the focal plane. With a selected channel in science mode, an optic is introduced into the corresponding guide channel to place an image of the telescope entrance pupil on the guide CCD. The starshade's lateral position is sensed from an image of the light distribution on the entrance pupil- thus the pixel resolution is given in centimeter in Table 1. At the entrance pupil, the starshade shadow has some structure, typically with a much diminished "spot of Arago" at the center. The lateral position of the telescope is sensed by imaging this structure and comparing with a library of expected images.

Figure 8 shows an image of the shadow structure in infrared light when the starshade is set up for visible science. The central dot appears directly on the line of sight between the center of the starshade and the star and thus forms the target for the guide system. Outside this core, there are two faint rings (there are predictable variations of the exact pattern between different starshades and starshade ranges) and then the flux increases with a monotonic slope towards the edge. 
Outside this region, a pattern reflective of the starshade geometry appears with, in this case, 24 peaks around a circumference, the modeled starshade having 24 petals. The central peak has a diameter $\sim$ range $\times \lambda /$ starshade diameter; in this case about $3 \mathrm{~m}$. The diameter of the smooth, sloping region is approximately $40 \mathrm{~m}$ and beyond this a pronounced pattern exists for about another $40 \mathrm{~m}$. The starshade GNC system (guidance navigation and control) utilizes this pattern to bring the starshade into line with the star when in the acquisition mode. Within the patterned region, the starshade follows the gradient down to the center. Once centered, the system maintains the central spot in the telescope pupil by periodically (every few $100 \mathrm{~s}$ ) firing thrusters on the starshade. This alignment system is facilitated by the brightness of the science target stars and the poor attenuation by the starshade in the guide bands. When a thruster firing occurs, science data taking is briefly suspended $(\sim 1 \mathrm{~s})$. This is because the thruster plumes are illuminated by the Sun and would contaminate the data. However, they rapidly dissipate and the flux at the detectors is small; the detectors are electronically cleared and resume data acquisition.

\subsection{Coronagraphs}

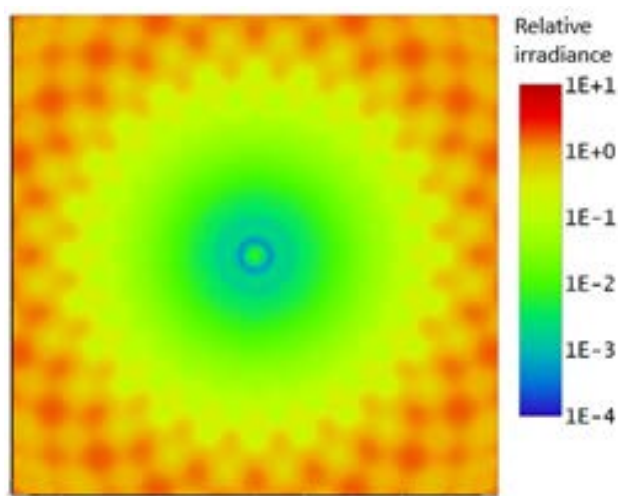

Figure 8: Starshade shadow at $1700 \mathrm{~nm}$ wavelength. The central spot, which is used for guiding when in science mode, has an intensity $\sim 7 e-3$ of the starlight. Away from the center, with the starshade misaligned, the gradient of the shadow is used to locate the direction of the center of the shadow.

With $20 \%$ bandwidth being the current state of the art for a high contrast coronagraph, covering the wavelength range 450 to $1,000 \mathrm{~nm}$ requires four observations. To reduce observation time, two coronagraphs are specified as shown schematically in Figure 9, covering the spectral ranges shown in Table 3. Light entering the coronagraphs is split into two bands, wavelengths shorter than $672 \mathrm{~nm}$ being passed to the "blue" channel and longer wavelengths to the "red" channel. Figure 10 shows the arrangement of the blue channel (the red channel is similar) and its component parts. A mechanical layout is shown in Figure 11. Within the two channels, dichroic filters set the optical bandwidth to $20 \%$, so that to cover the range requires two observations as seen in the table. Each channel carries a camera and an integral field spectrograph, selected by inserting a mirror. An additional infrared channel also resides on the red side and is selected similarly. It carries a slit spectrograph with $\mathrm{R}=40$ and covers the band 950-1800 $\mathrm{nm}$. An infrared avalanche diode detector is used on this channel. Two deformable mirrors (DMs) are used to correct the wavefront phase and amplitude. To obtain a compact layout, increasing stability and lowering the mass, it is desirable to use a small DM actuator spacing. For this reason, $64 \times 64$ actuator DMs of a commercially available type were specified with $0.4 \mathrm{~mm}$ actuator pitch yielding outer working angles (OWAs) shown in Table 3. The coronagraphs follow a similar layout to the WFIRST coronagraph ${ }^{6}$ design, while attempting to minimize the number of mirrors needed so as to maintain optical throughput. Following the common fine steering mirror, the red and blue channels are separated in collimated space

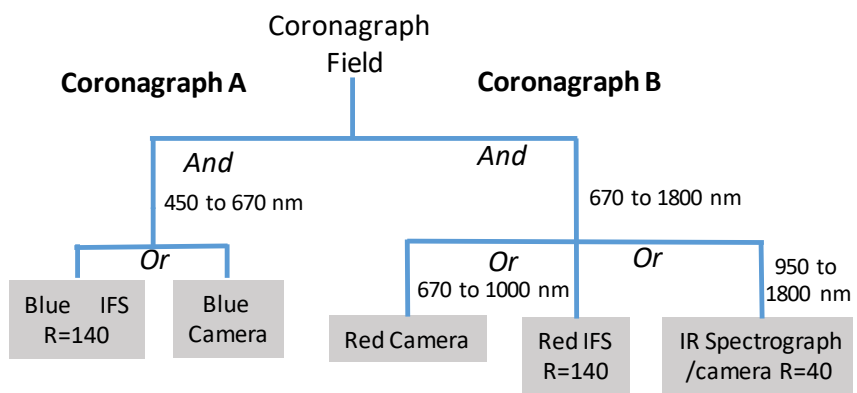

Figure 9: Coronagraph red and blue channels.

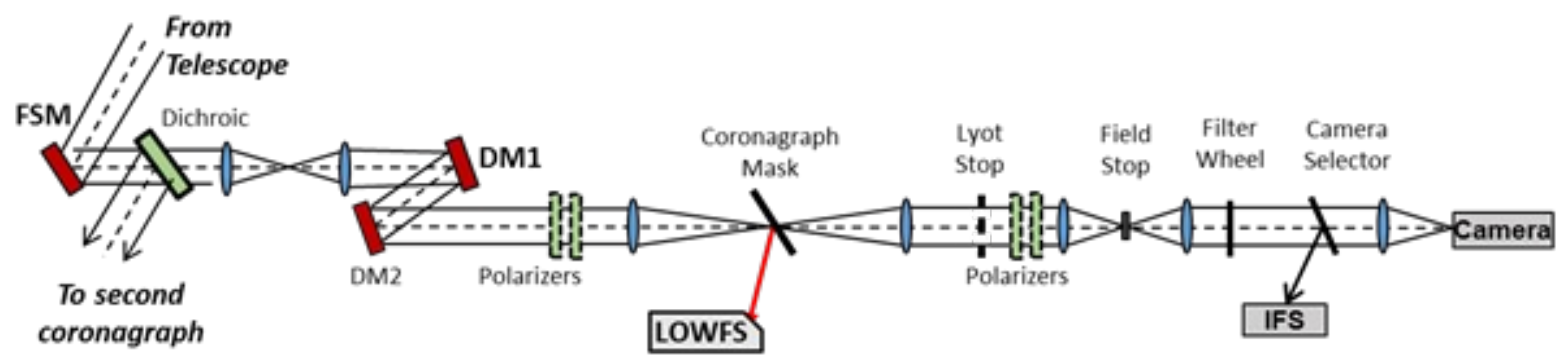

Figure 10: Simplified schematic layout of coronagraphs. 


\begin{tabular}{|c|c|c|c|}
\hline & "Blue" Channel & "Red" Channel & IR Channel \\
\hline \multicolumn{4}{|l|}{ Cameras } \\
\hline FOV & $1.5 "$ & $2.2^{\prime \prime}$ & 3.1" \\
\hline Wavelength bands & $\begin{array}{l}450-550 \mathrm{~nm} \\
550-672 \mathrm{~nm}\end{array}$ & $\begin{array}{c}672-821 \mathrm{~nm} \\
821-1,000 \mathrm{~nm}\end{array}$ & $950-1,800 \mathrm{~nm}$ \\
\hline Pixel resolution & $11.6 \mathrm{mas}$ & $17.3 \mathrm{mas}$ & 29.9 mas \\
\hline Telescope resolution & 23 mas (at $450 \mathrm{~nm}$ ) & 35 mas (at $672 \mathrm{~nm}$ ) & 49 mas (at $950 \mathrm{~nm}$ ) \\
\hline IWA (2.4 N/D) & 56 mas (at $450 \mathrm{~nm}$ ) & 83 mas (at $672 \mathrm{~nm}$ ) & 118 mas (at $950 \mathrm{~nm}$ ) \\
\hline OWA (as) & 0.74 & 1.11 & 1.57 \\
\hline Detector & $1 \times 1$ CCD201 & $1 \times 1$ CCD201 & $1 \times 1$ LMAPD \\
\hline Array width & 1024 & 1024 & $256 \times 320$ \\
\hline \multicolumn{4}{|l|}{ Spectrometers } \\
\hline FOV & $1.5 "$ & $2.2 "$ & 3.1" \\
\hline Spectrometer resolution $\lambda / \Delta \lambda$ & 140 & 140 & 40 \\
\hline Spectrometer type & IFS & IFS & Slit \\
\hline Detector & 1/4 CCD282 (EMCCD) & 1/4 CCD282 (EMCCD) & $1 \times 1$ LMAPD \\
\hline Array width (pixels) & 2048 & 2048 & $256 \times 320$ \\
\hline Deformable mirror & $64 \times 640.4 \mathrm{~mm}$ pitch & $64 \times 640.4 \mathrm{~mm}$ pitch & $64 \times 640.4 \mathrm{~mm}$ pitch \\
\hline
\end{tabular}

by a dichroic beam splitter. An initial relay of two off-axis parabolas (OAPs) sets the magnification to place the pupil on the DM. Telecentricity is not preserved at the entrance but is restored after the relay so that DM1 is positioned at a pupil plane. Following a fold, the beam strikes DM2 and is then focused onto the coronagraphic mask. Light reflected by the mask is directed to a low order wavefront sensor (LOWFS). Following recollimation of the science beam, it is apertured at the Lyot stop. After the Lyot stop the light can be directed via selector mirrors to the IFS, to the camera or in the case of the red channel, to the IR camera/spectrograph. Polarizers are included in the beam train to allow operation with existing vector vortex masks and also to allow selection of polarized light from the science targets, for example during disk imaging.

\section{Vortex and Hybrid Lyot Coronagraph Architectures}

Four main coronagraph families were initially considered: the shaped pupil (SP) and apodized pupil Lyot coronagraph (APLC), the phase-induced amplitude apodization complex mask coronagraph (PIAACMC), hybrid Lyot coronagraph ${ }^{7}$ and VVC ${ }^{8,9}$. The VVC family was found to present the most favorable trade-off between IWA and immunity to low-order aberrations. The VVC is a phase-based coronagraph that imprints a phase screw dislocation of the form $\mathrm{e}^{\mathrm{i} \theta}$ on the Airy diffraction pattern at the instrument focus, where $\theta$ is the azimuthal coordinate in the focal plane. When the star is centered on the phase ramp, the screw dislocation forced upon the electric field generates a singularity or optical vortex. While the phase undergoes rapid changes around the singularity, the field amplitude is zeroed out locally creating a dark hole. Upon propagation to the downstream Lyot stop (Figure 10), the dark hole grows to fill the entire pupil geometric area. The topological charge quantifies the number of times the vortex phase ramp goes through a full $2 \pi$ radian cycle. Mawet et al. ${ }^{8}$ demonstrated that perfect starlight rejection within the downstream geometric area can be achieved with an unobscured circular aperture and VVCs of even topological charges. Moreover, the topological charge can be seen as a knob allowing a trade-off of IWA for immunity to low order aberrations ${ }^{10,11}$. Indeed, the higher the charge, the lower the sensitivity to low-order aberrations, but the larger the IWA. The vortex coronagraph has been particularly popular on land-based adaptively corrected telescopes. Its small IWA, layout simplicity, intrinsic achromaticity, and high throughput makes it an

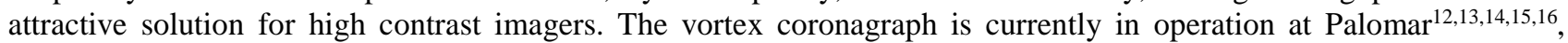
VLT $^{17,18}$, Subaru ${ }^{19}$, Keck ${ }^{20,21,22,23}$ and the Large Binocular Telescope ${ }^{24}$. For the HabEx $4 \mathrm{~m}$, topological charge 6 may represent the optimal tradeoff between inner working angle (2.4 $\lambda / \mathrm{D}$ at $50 \%$ total off-axis throughput), and immunity to low order aberrations: tip-tilt, defocus, astigmatism, coma, spherical. However, it does pass tertiary modes such as trefoil, which can be generated by the telescope mounts particularly on the primary mirror. Therefore a charge 8 vortex is also being considered and indeed, the coronagraph instruments could carry vortices of various charges and even alternative masks such as the well-developed Hybrid Lyot (HLC), considered as a backup due to its high technology readiness (TRL 5) and traceability to the WFIRST CGI. Both the HLC and VVC masks have a small reflective dot at the center of their 
masks that will reflect most of the starlight to the LOWFS. The LOWFS is used to detect wavefront error drift, primarily tilt and focus. Light passing through the mask is recollimated then passed through a Lyot stop to block light diffracted from the center of the mask. Finally, the light enters either a camera or an IFS where imaging and spectral measurements are completed.

Deformable Mirrors: The use of two DMs enables phase and amplitude control over both sides of the high contrast image, providing a large wellsuppressed dark region. There are currently two DM technologies suitable for HabEx. The first utilizes lead magnesium niobate (PMN) electrostrictive ceramic actuators on a 1 $\mathrm{mm}$ pitch to drive a continuous fusedsilica mirror face sheet. This technology is currently baselined on the WFIRST CGI. The second is based on microelectromechanical systems (MEMS) deformable mirror

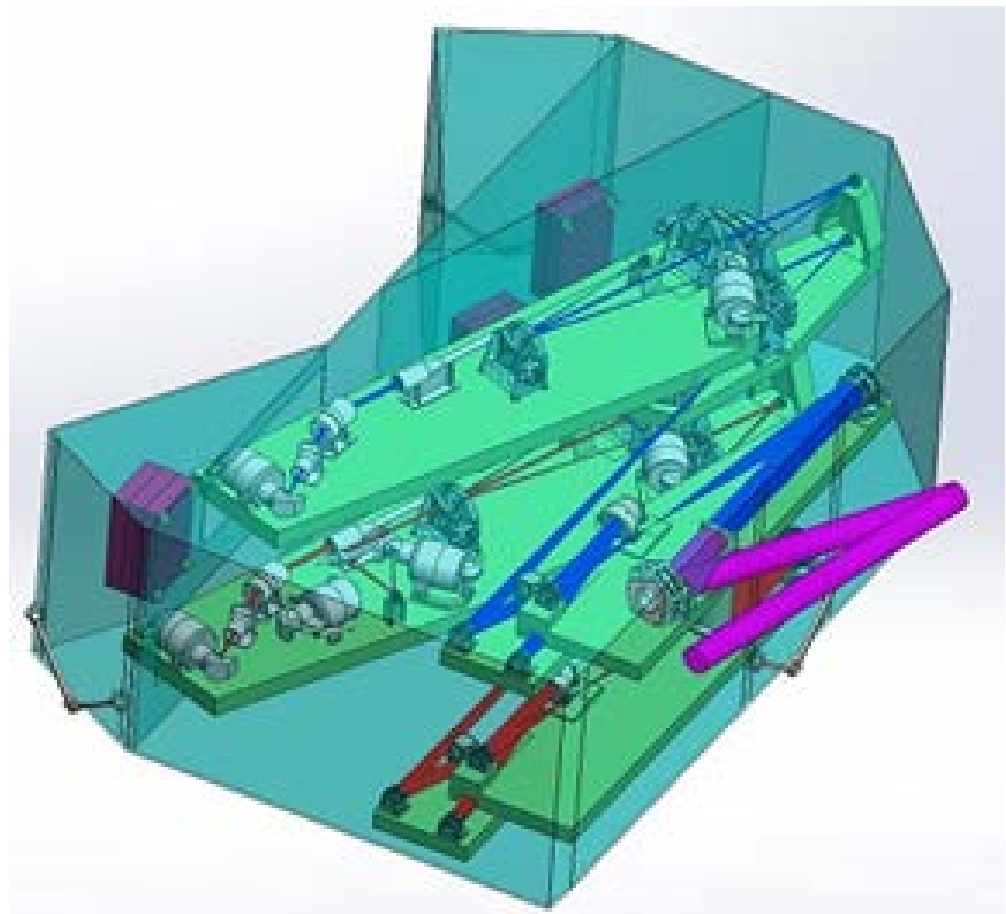

Figure 11: Showing the two coronagraph channels, blue and red. Light from the telescope enters center right- the purple rays are the beams from M3. technology. Each actuator in the MEMS

DM can be individually deflected by electrostatic actuation to achieve the desired pattern of deformation without hysteresis. The number of DM actuators determines the coronagraph OWA, while the pitch and number of actuators contribute to overall instrument size. HabEx has baselined a 64×64 MEMS DM with a $0.4 \mathrm{~mm}$ actuator pitch; the actuator count allows the coronagraph to reach a $32 \lambda / \mathrm{D}$ OWA, while the small actuator pitch helps minimize overall instrument size. Simulations show that the DM configuration is sufficient to provide a capability in wavefront control in both amplitude and phase domains, correcting minute wavefront errors due to fabrication and alignment inaccuracies in the system and enabling the required deep $\left(10^{-10}\right)$ starlight suppression for this instrument.

Coronagraphic Masks: To cover the optical bands within both the visible and infrared channels, multiple masks are needed to provide the required starlight suppression. These masks are carried by a wheel mechanism, with the appropriate mask rotated into position depending on the science waveband selected for observation. Both the HLC and the VVC masks are tilted slightly away from the beam path and each type of mask has a reflective spot at the mask center, sending incident starlight into the LOWFS.

Lyot Stops: The Lyot stop design for the vortex coronagraph does not depend on wavelength, and so in principle, a single Lyot stop is needed. The HLC requires a series of Lyot stops optimized for each waveband, so a Lyot stop wheel would be required.

Low Order Wavefront Sensor: The LOWFS sensor is a Zernike wavefront sensor $\left(Z_{W F S}^{25}\right)$ based on the Zernike phase contrasting principle where a small phase step (either a dimple or a raised area) with phase difference of $\sim \pi / 2$ is placed at center of (in this case) the vortex mask. Light reflecting from the dimple and the surrounding area interfere to produce intensity variations in a pupil image formed on the LOWFS camera. Thus the LOWFS uses the rejected starlight from coronagraph to sense low order wavefront error, including pointing error and thermally induced low order wavefront drift. The sensed tip tilt error is used to control the FSM for fine corrections to the line of sight. Accumulated larger pointing error is fed to the spacecraft system and corrected using the microthrusters.

Focal Planes: Detector arrays for the visible channels are EMCCD types, selected because of their exceptionally low effective read noise. The imaging focal planes (blue and red channels) consist of a single EMCCD per channel operated at 153K. The chosen type is a modified CCD201 with delta doping and a thickened substrate together with a broadband "astro" coating giving response out to 1,000 nm. Pixel scale is as shown in Table 3 . The corresponding IFS focal planes 
consists of a modified, cut down version of CCD282 which is a $4 \mathrm{k} \times 4 \mathrm{k}$ device with a $2 \mathrm{k} \times 4 \mathrm{k}$ frame store at each end. The device format allows it to be cut into quarters forming a $2 \mathrm{k} \times 2 \mathrm{k}$ sensor area with a $2 \mathrm{k} \times 2 \mathrm{k}$ frame store. The device would be operated at $163 \mathrm{~K}$ to minimize dark current. For the infrared, an HgCdTe avalanche photodiode array (Saphira LMAPD) is baselined. The detector is cooled to $60 \mathrm{~K}$ to minimize dark current. It has a low read noise with avalanche gains of 50 or more available.

Filters: The coronagraphs carry a set of bandpass filters as shown in Table 4 . Set $1-5$ covers the passbands of the vortex masks and will be employed when the spectrographs are in use. Set $6-8$ is close to the wavelengths put forward by Krissansen-Totton et al. ${ }^{26}$ as being ideal for identifying earthlike exoplanets. Set $9-13$ is for giant planet characterization and the remaining three cover the passbands of the infrared coronagraph.

\section{SUMMARY}

With an unobscured $4 \mathrm{~m}$ diameter aperture, the baseline HabEx 4 m observatory would be capable of collecting three times as many photons as the $2.4 \mathrm{~m}$ Hubble Space Telescope. Its diffraction resolution limit would be 21 milliarcseconds at $400 \mathrm{~nm}$, compared to HST's performance of 34.4 mas. HabEx is designed to be the most stable astronomical observing platform ever; capable of relative pointing stability to 0.7 mas. HST's best pointing stability is $\sim 2$ mas. Furthermore, this design concept is based solely on manufacturing capabilities and state of the art telescope technologies presently available.

The baseline HabEx mission concept is composed of two separate spacecraft flying in formation in a Sun-Earth L2 orbit. One spacecraft carries a $4 \mathrm{~m}$ off-axis telescope, while the other spacecraft carries a $72 \mathrm{~m}$ starshade. Together, when flying in formation, they form an externally occulting observatory for exoplanet imaging and spectral characterization. Using just the telescope and its internal coronagraph, a second method of detecting and characterizing exoplanets is available. The starshade suppresses the light from the target star while the telescope's starshade imager observes the planetary system surrounding the target star. To form this observatory, the starshade positions into the line-of-sight (LOS) between the telescope and the target star at an approximate 124,000 km separation from the telescope, and maintains alignment using a positional control loop carried over a spacecraft-to-spacecraft radio link. Position sensing is carried out by instrumentation on the telescope spacecraft and position control is handled by the propulsion system on the starshade spacecraft. The primary mission would run for five years, but the telescope includes enough fuel to continue operations for five additional years. The starshade has fuel for five years of operations after which it can no longer slew to new targets and must hold the L2 orbit until serviced. Serviceability is a requirement for all large astrophysics observatories; both the starshade and the telescope are able to be refueled and upgraded, however, the starshade occulter cannot be replaced during servicing.

The greatest scope of exoplanet science observations is accomplished using both the internal coronagraph and the starshade working in concert. These two instruments are complementary in nature. While the starshade instrument is capable of efficient high-contrast imaging and spectroscopy over a large field of view, it is limited in the number of observations due to the large slew times of the starshade occulter. The coronagraph, on the other hand, is capable of faster slews, making many more observations possible, but has a narrower, high-contrast FOV with reduced spectrographic capability. Working together, the starshade and coronagraph can achieve the repeated planet detections required to determine orbits and the high-resolution spectral profiles needed to characterize exoplanet atmospheric gases. 
To ensure coronagraph-driven LOS pointing and wavefront stability, the telescope includes precision thermal control, mirror positional control using laser metrology, and a fine-guiding sensor (FGS) paired with microthrusters for pointing control during observations. Two on-board general astrophysics instruments, HWC and UVS are provided and enable general science observations and high resolution spectroscopy from $115 \mathrm{~nm}$ through to $1800 \mathrm{~nm}$.

\section{ACKNOWLEDGEMENTS}

The information in this paper on the HabEx concept is provided prior to any decisions or commitments being made and is provided for planning and discussion purposes only. This work was conducted at the Jet Propulsion Laboratory, California Institute of Technology, under contract with the National Aeronautics and Space Administration. Copyright 2018 California Institute of Technology. Government sponsorship acknowledged. All rights reserved. URS\#274214.

\section{REFERENCES}

[1] Stefan Martin, Mayer Rud, Paul Scowen, Daniel Stern, Joel Nissen, John Krist, "HabEx space telescope optical system”, Proc. SPIE. 10398, 5 September 2017.

[2] Paul Scowen, Daniel Stern, Rachel Somerville, Mayer Rud, Stefan Martin, Matthew Beasley, "Science and architecture drivers for the HabEx Ultraviolet Spectrograph (UVS)”, Proc. SPIE. 10398, 5 September 2017.

[3] Michael McElwain, Avi Mandell, Qian Gong, Jorge Llop-Sayson, Timothy Brandt, Victor Chambers, Bryan Grammer, Bradford Greeley, George Hilton, Marshall Perrin, Karl Stapelfeldt, Richard Demers, Hong Tang, Eric Cady, "PISCES: an integral field spectrograph technology demonstration for the WFIRST coronagraph", Proc. SPIE. 9904, 29 July 2016.

[4] Nikzad, Shouleh, Michael E Hoenk, Frank Greer, Blake Jacquot, Steve Monacos, Todd J Jones, Jordana Blacksberg, Erika Hamden, David Schiminovich, and Chris Martin. "Delta-doped electron-multiplied CCD with absolute quantum efficiency over $50 \%$ in the near to far ultraviolet range for single photon counting applications.” Applied Optics 51 (3):365-369, 2012.

[5] Manan Arya, David Webb, James McGown, P. Douglas Lisman, Stuart Shaklan, S. Case Bradford, John Steeves, Evan Hilgemann, Brian Trease, Mark Thomson, Steve Warwick, Gregg Freebury, Jamie Gull, "Starshade mechanical design for the Habitable Exoplanet imaging mission concept (HabEx)”, Proc. SPIE. 10400, 12 September 2017

[6] Trauger, John T, Dwight C Moody, John E Krist, and Brian L Gordon, "Hybrid Lyot coronagraph for WFIRSTAFTA: coronagraph design and performance metrics.” Journal of Astronomical Telescopes, Instruments, and Systems 2 (1):011013, 2016.

[7] M. Charley Noecker, Feng Zhao, Rick Demers, John Trauger, Olivier Guyon, N. Jeremy Kasdin, “Coronagraph instrument for WFIRST-AFTA", Journal of Astronomical Telescopes, Instruments, and Systems 2 (1).| 11 March 2016

[8] Mawet, D, Pierre Riaud, Olivier Absil, and Jean Surdej. 2005. “Annular groove phase mask coronagraph.” The Astrophysical Journal 633 (2):1191.

[9] Foo, Gregory, David M Palacios, and Grover A Swartzlander. 2005. “Optical vortex coronagraph.” Optics letters 30 (24):3308-3310.

[10] Mawet, Dimitri, Laurent Pueyo, Dwight Moody, John Krist, and Eugene Serabyn. 2010. "The Vector Vortex Coronagraph: sensitivity to central obscuration, low-order aberrations, chromaticism, and polarization.” Modern Technologies in Space-and Ground-based Telescopes and Instrumentation.

[11] Ruane, G, D Mawet, J Kastner, T Meshkat, M Bottom, B Femenia Castella, Olivier Absil, C Gomez Gonzalez, Elsa Huby, and Z Zhu. "Deep imaging search for planets forming in the TW Hya protoplanetary disk with the Keck/NIRC2 vortex coronagraph.” The Astronomical Journal 154 (2):73. 2017.

[12] Serabyn, E, D Mawet, and R Burruss. "An image of an exoplanet separated by two diffraction beamwidths from a star.” Nature 464 (7291):1018. 2010.

[13] Mawet, D, E Serabyn, K Liewer, R Burruss, J Hickey, and D Shemo. 2010. "The Vector Vortex Coronagraph: Laboratory Results and First Light at Palomar Observatory.” Astrophysical Journal 709 (1):53-57. 2010.

[14] Mawet, Dimitri, Eugene Serabyn, J Kent Wallace, and Laurent Pueyo. 2011. "Improved high-contrast imaging with on-axis telescopes using a multistage vortex coronagraph.” Optics letters 36 (8):1506-1508. 
[15]Bottom, Michael, Stefan Martin, Carl Seubert, Eric Cady, Shannon Kian Zareh, and Stuart Shaklan. 2017. "Precise starshade stationkeeping and pointing with a Zernike wavefront sensor.” Techniques and Instrumentation for Detection of Exoplanets VIII. Proc. SPIE. 10400, 5 September 2017.

[16] Bottom, Michael, J Chris Shelton, James K Wallace, Randall Bartos, Jonas Kuhn, Dimitri Mawet, Bertrand Mennesson, Rick Burruss, and Eugene Serabyn. 2016. "Stellar double coronagraph: a multistage coronagraphic platform at Palomar observatory.” Publications of the Astronomical Society of the Pacific 128 (965):075003. 2016.

[17] Mawet, Dimitri, Laurent Pueyo, Alexis Carlotti, Bertrand Mennesson, Eugene Serabyn, and James K Wallace. 2013. "Ring-apodized vortex coronagraphs for obscured telescopes. I. Transmissive ring apodizers." The Astrophysical Journal Supplement Series 209 (1):7. 2013.

[18] Absil, Olivier, Julien Milli, Dimitri Mawet, A-M Lagrange, Julien Girard, Gaël Chauvin, Anthony Boccaletti, Christian Delacroix, and Jean Surdej. 2013. "Searching for companions down to 2 AU from $\beta$ Pictoris using the L'band AGPM coronagraph on VLT/NACO.” Astronomy \& Astrophysics 559:L12.

[19]Kühn, Jonas, Olivier Guyon, Julien Lozi, Tomoyuki Kudo, Nemanja Jovanovic, Garima Singh, Eugene Serabyn, Frantz Martinache, Motohide Tamura, and Denis Defrère. 2017. An H-band Vector Vortex Coronagraph for the Subaru Coronagraphic Extreme-Adaptive Optics System. 2017.

[20] Absil, Olivier, Dimitri Mawet, Mikael Karlsson, Brunella Carlomagno, Valentin Christiaens, Denis Defrère, Christian Delacroix, Bruno Femenía Castella, Pontus Forsberg, and Julien Girard. 2016. "Three years of harvest with the vector vortex coronagraph in the thermal infrared.” Ground-based and Airborne Instrumentation for Astronomy VI.

[21] Serabyn, Eugene, Elsa Huby, Keith Matthews, Dimitri Mawet, Olivier Absil, Bruno Femenia, Peter Wizinowich, Mikael Karlsson, Michael Bottom, and Randy Campbell. 2017. "The WM Keck Observatory Infrared Vortex Coronagraph and a First Image of HIP 79124 B.” The Astronomical Journal 153 (1):43.

[22] Mawet, Dimitri, Garreth Ruane, Wenhao Xuan, Daniel Echeverri, Nikita Klimovich, Michael Randolph, Jason Fucik, James K Wallace, Ji Wang, and Gautam Vasisht. "Observing exoplanets with high-dispersion coronagraphy. II. Demonstration of an active single-mode fiber injection unit.” The Astrophysical Journal 838 (2):92. 2017.

[23] Reggiani, Maddalena, and VORTEX TEAM. 2017. "A candidate accreting protoplanet in the transition disk around MWC 758."

[24]Defrere, Denis, Olivier Absil, P Hinz, J Kuhn, D Mawet, B Mennesson, A Skemer, K Wallace, V Bailey, and E Downey. 2014. "L'-band AGPM vector vortex coronagraph's first light on LBTI/LMIRCam.” Adaptive Optics Systems IV.

[25] J. Kent Wallace, Shanti Rao, Rebecca Jensen-Clem, Gene Serabyn, "Phase-shifting Zernike interferometer wavefront sensor” Proc. SPIE. 8126, 27 September 2011.

[26] Krissansen-Totton, J., E. W. Schwieterman, B. Charnay, G. Arney, T. D. Robinson, V. Meadows, D. C. Catling (2016). Is the Pale Blue Dot unique? Optimized photometric bands for identifying Earth-like exoplanets. The Astrophysical Journal 817 (1), 31. 